\title{
Pulmonary thromboembolism post-COVID convalescent plasma therapy: adding fuel to a smoldering fire!
}

\section{To the Editor}

The current coronavirus disease 2019 (COVID-19) pandemic has caused millions of cases worldwide and is still creating havoc all around the world. The novel coronavirus mainly affects the lungs and causes severe disease in the form of acute respiratory distress syndrome. These patients are at an additional risk of developing pulmonary thromboembolism owing to multiple mechanisms such as endothelial injury, coagulopathy, and vasculopathy due to virus related damage [1]. COVID convalescent plasma is presently being used extensively in the treatment of severe cases [2, 3]. It has shown promising results in COVID-19 cases, but has an additional risk factor of being highly prothrombotic which predisposes these patients to pulmonary thromboembolism $[4,5]$. This has rarely been reported in literature. Herein, we present a case of a patient with severe COVID-19 disease who developed pulmonary thromboembolism post-convalescent plasma administration while he was on a therapeutic dose of heparin.

A 60-year-old male, who had also been diagnosed with primary hypertension for 5 years (well-controlled on oral antihypertensives), bronchial asthma for 30 years (well-controlled on inhaled bronchodilators and inhaled corticosteroids), and benign prostatic hyperplasia for 5 years (treated with oral alpha-blockers), reported with a history of moderate grade fever and cough of 4 days duration. He also complained of dyspnoea on rest that had been present for 1 day. He was diagnosed with COVID-19 infection after a nasopharyngeal throat swab and reverse transcriptase-polymerase chain reaction (RT-PCR) testing. At presentation, he was hypoxic on room air (80\%), had a $\mathrm{pO}_{2} / \mathrm{FiO}_{2}<100$, and was managed via high flow nasal cannula (flow $60 \mathrm{~L} / \mathrm{min}$ and $\left.\mathrm{FiO}_{2}-100 \%\right)$. His chest roentgenogram showed bilateral peripheral air-space opacities involving bilateral mid- and lower zones (Figure 1). He was concurrently initiated on injectable steroids (IV methylprednisolone, $1 \mathrm{mg} / \mathrm{kg}$ twice daily), antibiotics (iv ceftriaxone and levofloxacin), and a therapeutic dose of low-molecular-weight heparin (60 mg subcutaneously, twice daily). He had further deterioration and he was diagnosed as having cytokine storm syndrome based on increased inflammatory markers (C-reactive protein, ferritin, D-dimer, lactate dehydrogenase, and interleukin-6) and an increased requirement for oxygen. We managed him with 2 doses of an anti-IL6 agent (tocilizum$\mathrm{ab}, 8 \mathrm{mg} / \mathrm{kg}$ ) and 2 doses of COVID convalescent plasma (CCP) at $200 \mathrm{ml}$ per dose. He showed a significant response with improvement in clinical, radiological, and lab parameters. However, after 12 hours of CCP therapy, he showed deterioration with an increased requirement for oxygen. He was hypoxic and it was becoming difficult to wean him off oxygen. In view of a suspicion of pulmonary thromboembolism and a raised D-dimer level ( $>20 \mathrm{ng} / \mathrm{mL})$, he underwent a computed tomography pulmonary angiogram which showed subsegmental thrombi in the right lower lobe and segmental thrombi in the left upper lobe which confirmed our suspicion (Figure 2). Since he was normotensive, he was continued on therapeutic heparin and later discharged on oral anti-coagulants. Currently,

Address for correspondence: Robin Choudhary, Army Institute of Cardiothoracic Science, Armed Forces Medical College, Pune, India; e-mail: robinch19@gmail.com DOI: 10.5603/ARM.a2021.0022

Received: 30.11 .2020

Copyright (C) 2021 PTChP

ISSN 2451-4934 


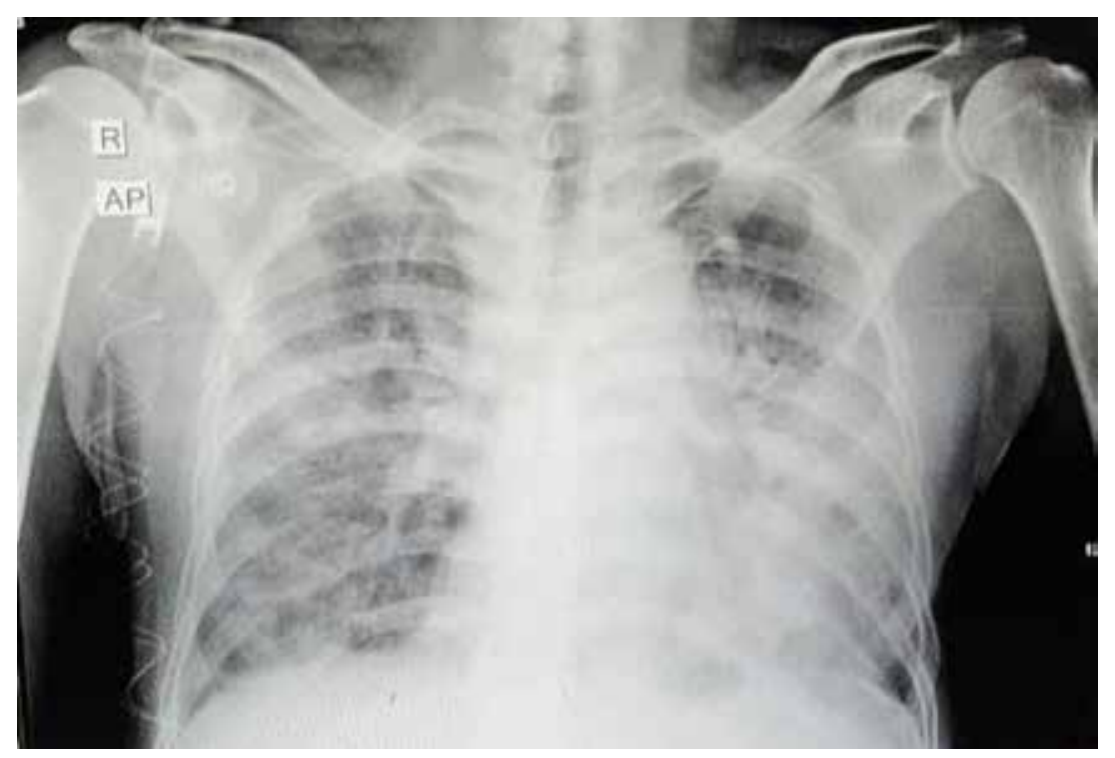

Figure 1. Chest skiagram showing bilateral mid- and lower zone airspace opacities

his condition has improved and he is able to maintain saturation on room air.

The present COVID-19 pandemic has been implicated in causing an immense prothrombotic state in affected patients which can cause an increased risk of pulmonary thromboembolism even when they are on prophylactic LMWH [1]. The pathophysiology includes endothelial inflammation, complement activation, thrombin generation, and platelet and leukocyte recruitment. These patients warrant prophylactic heparin to prevent this catastrophe [1]. COVID convalescent plasma has emerged as a potential therapy for severe COVID-19 patients [2-4]. How- ever these patients are at a higher risk of pulmonary thromboembolism post COVID convalescent plasma therapy, which is rarely reported. These patients are already in a dysregulated coagulative state and the administration of convalescent plasma can tip the balance towards a catastrophic event like pulmonary thromboembolism $[5,6]$. Thus COVID-19 convalescent plasma therapy warrants a more rigorous clinical trial to assess its safety profile.

\section{Conflict of interest}

Non declared.

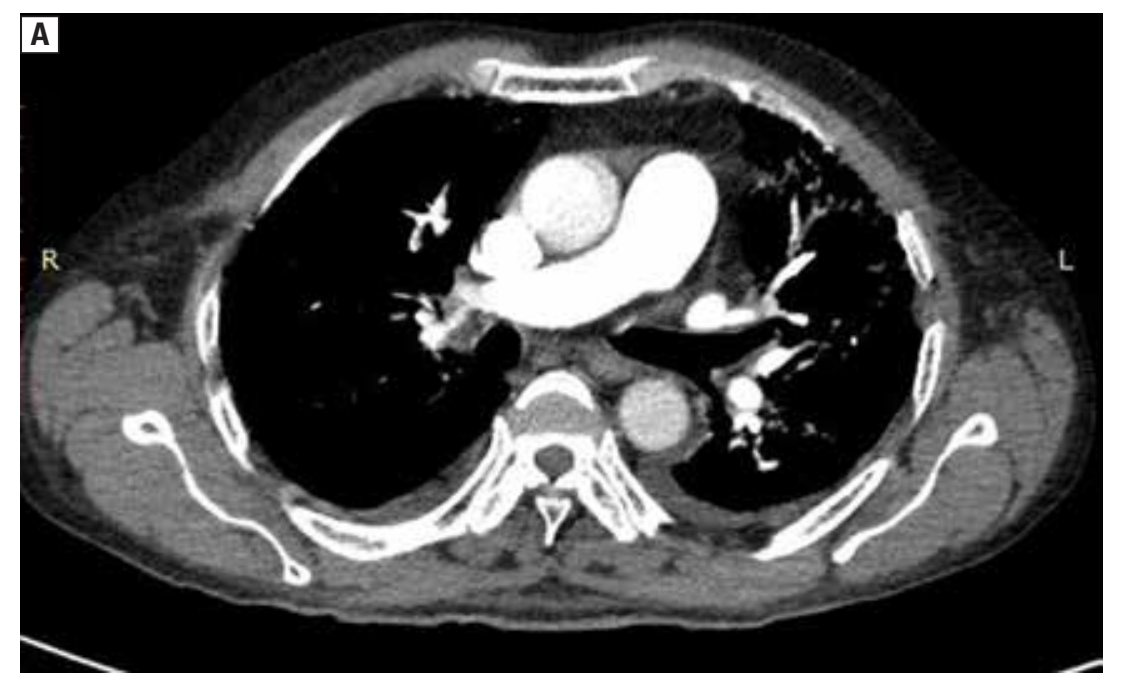

Figure 2. A. Computed tomography of pulmonary angiogram showing subsegmental thrombi in the right lower lobe and segmental thrombi in the left upper lobe 

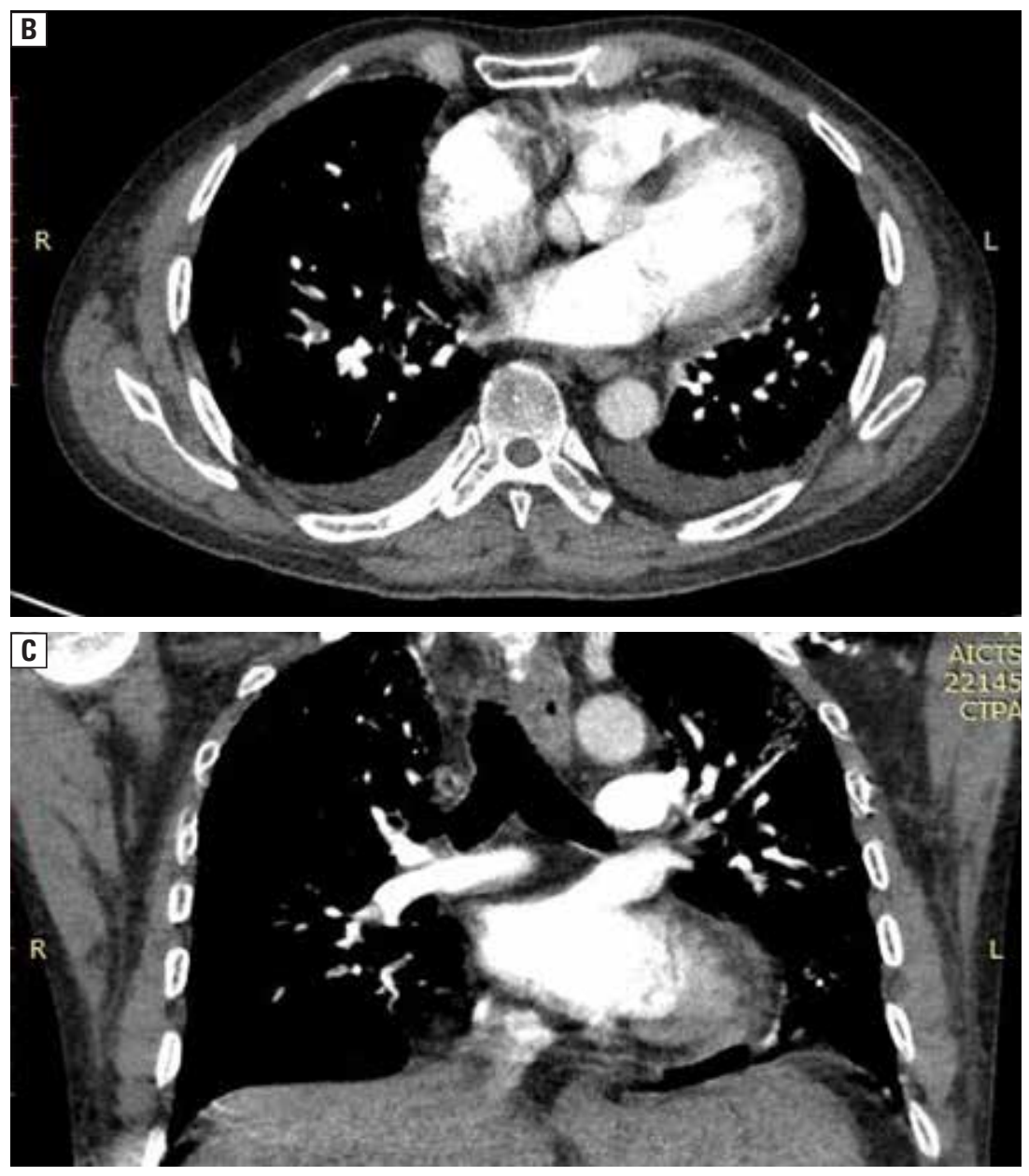

Figure 2. B-C. Computed tomography of pulmonary angiogram showing subsegmental thrombi in the right lower lobe and segmental thrombi in the left upper lobe

\section{References:}

1. Sakr Y, Giovini M, Leone M, et al. Pulmonary embolism in patients with coronavirus disease-2019 (COVID-19) pneumonia: a narrative review. Ann Intensive Care. 2020; 10(1): 124, doi: 10.1186/s13613-020-00741-0, indexed in Pubmed: 32936400.

2. Focosi D, Anderson AO, Tang JW, et al. Convalescent plasma therapy for COVID-19: state of the art. Clin Microbiol Rev. 2020; 12(33): 4791-4797.

3. Yiğenoğlu TN, Hacıbekiroğlu T, Berber İ, et al. Convalescent plasma therapy in patients with COVID-1. J Clin Apher. 2020; 35(4): 367-373, doi: http://www.ncbi.nlm.nih.gov/ pubmed/32643200.
4. Joyner MJ, Wright RS, Fairweather D, et al. Early safety indicators of COVID-19 convalescent plasma in 5000 patients. J Clin Invest. 2020; 130(9): 4791-4797, doi: 10.1172/JCI140200, indexed in Pubmed: $\underline{32525844}$.

5. Sanfilippo F, La Rosa V, Oliveri F, et al. Convalescent plasma for COVID-19: the risk of pulmonary embolism should not be underestimated! Crit Care . 2020; 24(1): 531.

6. Nair PM, Rendo MJ, Reddoch-Cardenas KM, et al. Recent advances in use of fresh frozen plasma, cryoprecipitate, immunoglobulins, and clotting factors for transfusion support in patients with hematologic disease. Semin Hematol. 2020; 57(2): 73-82, doi: $10.1053 /$ j.seminhematol.2020.07.006, indexed in Pubmed: $\underline{32892846}$. 